\title{
Coaticook : Analyse scientifique de la vulnérabilité intégrant l'implication des acteurs locaux et citoyens pour une ville et une communauté plus résiliente
}

\section{Coaticook: Scientific analysis of vulnerability integrating implication of local stakeholders and citizens for a more resilient city and community}

\author{
Isabelle Thomas ${ }^{1}$, Alexandre Gagnon ${ }^{2}$ \\ ${ }^{1}$ École d'urbanisme et d'architecture de paysage de l'Université de Montréal, professeure titulaire, \\ isabelle.thomas.1@umontreal.ca \\ ${ }^{2}$ École d'urbanisme et d'architecture de paysage de l'Université de Montréal, auxiliaire de recherche, \\ agagnon01@hotmail.com
}

RÉSUMÉ. Le ruisseau Pratt a inondé plusieurs fois le centre-ville de Coaticook et causé de graves dommages, notamment en 2014 et 2015. Afin d'améliorer la compréhension du risque et cheminer vers une meilleure gestion, adaptation et résilience, la vulnérabilité de la population et du territoire est cartographiée et analysée par le Groupe d'étude sur la capacité d'adaptation et la résilience (GECAR), affilié à l'Université de Montréal, à l'aide d'une méthode d'analyse développée en collaboration avec le ministère de la Sécurité publique du Québec (MSP). Une analyse des dommages est également réalisée. De plus, la méthode d'analyse intègre l'implication des acteurs locaux et les citoyens afin de recueillir la connaissance locale et de progresser vers des stratégies et actions réalisables, acceptées et adaptées au contexte pour renforcer la résilience à Coaticook. La démarche itérative de recherche conçoit la résilience comme un processus dynamique, adaptatif et souple et s'inscrit dans une volonté de conscientisation au risque et dans un réel changement de paradigme : s'adapter en allant au-delà du retour à la normale.

ABSTRACT. Pratt stream flooded downtown Coaticook several times and caused serious damage, particularly in 2014 and 2015. To improve understanding of risk and move towards better management, adaptation and resilience, the vulnerability of the population and the territory is mapped and analyzed by the Groupe d'étude sur la capacité d'adaptation et la résilience (GECAR), affiliated with the University of Montreal, using an analysis method developed in collaboration with the Quebec ministry of public Security (MPS). A damage analysis is also performed. In addition, the analysis method integrates the involvement of local stakeholders and citizens in order to gather local knowledge and move towards achievable, accepted and context-specific strategies and actions to build resilience in Coaticook. The iterative approach conceives resilience as a dynamic, adaptive and flexible process and is part of a commitment to risk awareness and a real paradigm shift: adapt by going beyond the return to normality.

MOTS-CLÉS. Résilience, Vulnérabilité, Adaptation, SIG, Implication, Collaboration.

KEYWORDS. Resilience, Vulnerability, Adaptation, GIS, Implication, Collaboration. 


\section{Introduction : la compréhension et l'évaluation du risque}

Les inondations au Québec constituent un risque naturel important et une menace réelle aux populations. Ce risque est appelé à évoluer et à se transformer dans un contexte de changements climatiques et d'urbanisation. Il faut donc travailler dès maintenant à une meilleure compréhension du phénomène inondation et de ses conséquences, dans l'optique de préconiser un renouvellement des approches en prévention des risques pour soutenir une société et un territoire résilients (Ouranos, 2018). Si la connaissance de l'aléa est tout à fait primordiale, il est aussi important de s'attarder aux facteurs sociaux, physiques et institutionnels qui influencent la vulnérabilité face aux événements ainsi que la capacité d'adaptation (Thomas, Bleau et coll., 2012). Pour ce faire, la cartographie de la vulnérabilité représente un outil incontournable facilitant la compréhension du risque, la sensibilisation et la planification du territoire et constituant un outil de premier plan dans l'aide à la décision (Ouranos, 2018). Cette démarche de recherche doit s'intégrer dans un effort commun avec les organismes, experts locaux et citoyens afin de tenir compte de la réalité du terrain et identifier des solutions durables, acceptées et adaptées au contexte (Tricot, 2008).

Avec l'objectif d'améliorer à court, moyen et long terme la gestion du risque et de renforcer la capacité d'adaptation et la résilience du territoire et des communautés, le Groupe d'étude sur la capacité d'adaptation et la résilience (GECAR), affilié à l'Université de Montréal, met en œuvre une démarche de recherche innovante. Celle-ci permet à la fois de connaître et d'analyser la vulnérabilité tout en faisant participer les acteurs locaux à la démarche. Elle reflète une vision de la résilience conçue comme un processus dynamique, adaptatif et souple, qui englobe plusieurs stratégies dont la conscientisation progressive au risque et la transformation de la fabrique de la ville, à toutes les échelles spatiales et temporelles (Mairie de Paris, 2017).

En 2014 et 2015, le ruisseau Pratt a inondé le centre-ville de Coaticook et a causé de graves dommages. À la suite de ces événements, afin d'assurer la sécurité des citoyens et protéger le milieu bâti en améliorant la gestion du risque, la Ville de Coaticook a sollicité le ministère de la Sécurité publique du Québec (MSP) pour bénéficier du Cadre pour la prévention de sinistres 2013-2022, un programme générant des investissements en appréciation, traitement et communication des risques (MSP, 2018). La ville de Coaticook, avec cette sollicitation, souhaitait notamment obtenir un soutien financier pour la construction d'un ou de plusieurs bassins de rétention. Avant d'accorder ou non les fonds, le risque relié aux inondations du ruisseau Pratt devait être documenté, soit à la fois l'aléa et la vulnérabilité.

Dans ce contexte, le GECAR a été mandaté ${ }^{1}$ par le MSP pour évaluer la vulnérabilité reliée aux inondations causées par le ruisseau Pratt à Coaticook. En parallèle, une équipe de l'Université Laval menée par Brian Morse ${ }^{2}$ s'est concentrée sur l'analyse de l'aléa.

De manière plus exhaustive, le GECAR a appliqué sa méthode d'analyse de la vulnérabilité, développée en collaboration avec le MSP depuis 2016 avec une étude sur la vulnérabilité aux inondations menée dans la ville de Saint-Raymond, en y intégrant des séances de présentation et de discussion de résultats et une table participative avec les acteurs locaux. Une analyse des dommages est également réalisée tout comme un questionnaire aux citoyens, celui-ci de manière complémentaire afin d'aborder la perception du risque. Tous ces éléments ont été bonifiés de manière itérative grâce à l'implication des acteurs locaux et des citoyens, permettant une plus grande richesse d'analyse et un ancrage solide dans le contexte.

\footnotetext{
${ }^{1}$ Cette recherche a été appuyée par une contribution financière de 45000 \$ provenant du Cadre pour la prévention de sinistres 2013-2022 du gouvernement du Québec [CPS 16-17-16]
}

\footnotetext{
${ }^{2}$ Ingénieur et professeur titulaire au Département de génie civil et de génie des eaux de l'Université Laval
} 


\section{Contexte géographique : la ville de Coaticook aux prises avec un ruisseau capricieux}

La ville de Coaticook, située à environ 30 kilomètres au sud de la ville de Sherbrooke, est la plus grande ville de la MRC (municipalité régionale de comté) du même nom et est traversée dans sa zone urbaine (voir figure 1) par la rivière Coaticook qui coule vers le nord-ouest.

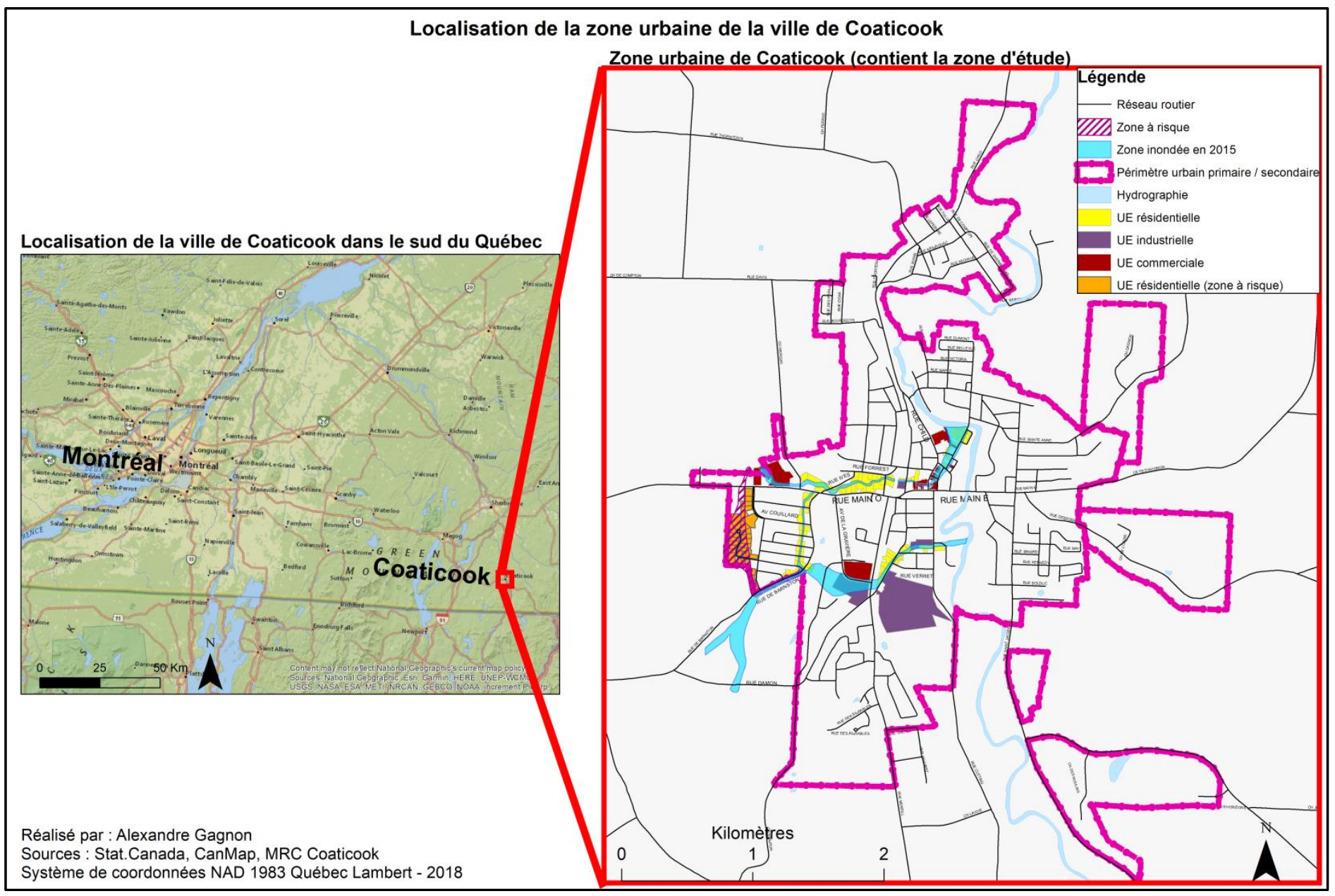

Figure 1. Localisation de la zone urbaine de la ville de Coaticook. Auteur : A. Gagnon, 2018

La figure 2 permet de mieux visualiser la zone d'étude pour la recherche, située dans la zone urbaine de la ville de Coaticook, qui comprend notamment la zone inondée en 2015, la zone à risque et les unités d'évaluation ${ }^{3}$ en contact avec le polygone de la zone inondée. La zone à risque est une zone où il n'y a pas eu de débordement notable en 2015, mais qui serait susceptible d'être inondée si les cours d'eau se comportaient différemment lors d'une prochaine inondation. Les unités d'évaluation en contact avec la zone inondée n'ont pas toutes subi des dommages, mais permettent d'illustrer le nombre important de terrains affectés et les usages du sol concernés.

\footnotetext{
${ }^{3}$ Une unité d'évaluation est un immeuble ou un regroupement d'immeubles adjacents appartenant à un même propriétaire et utilisés à une même fin prédominante (MAMH, 2010).
} 


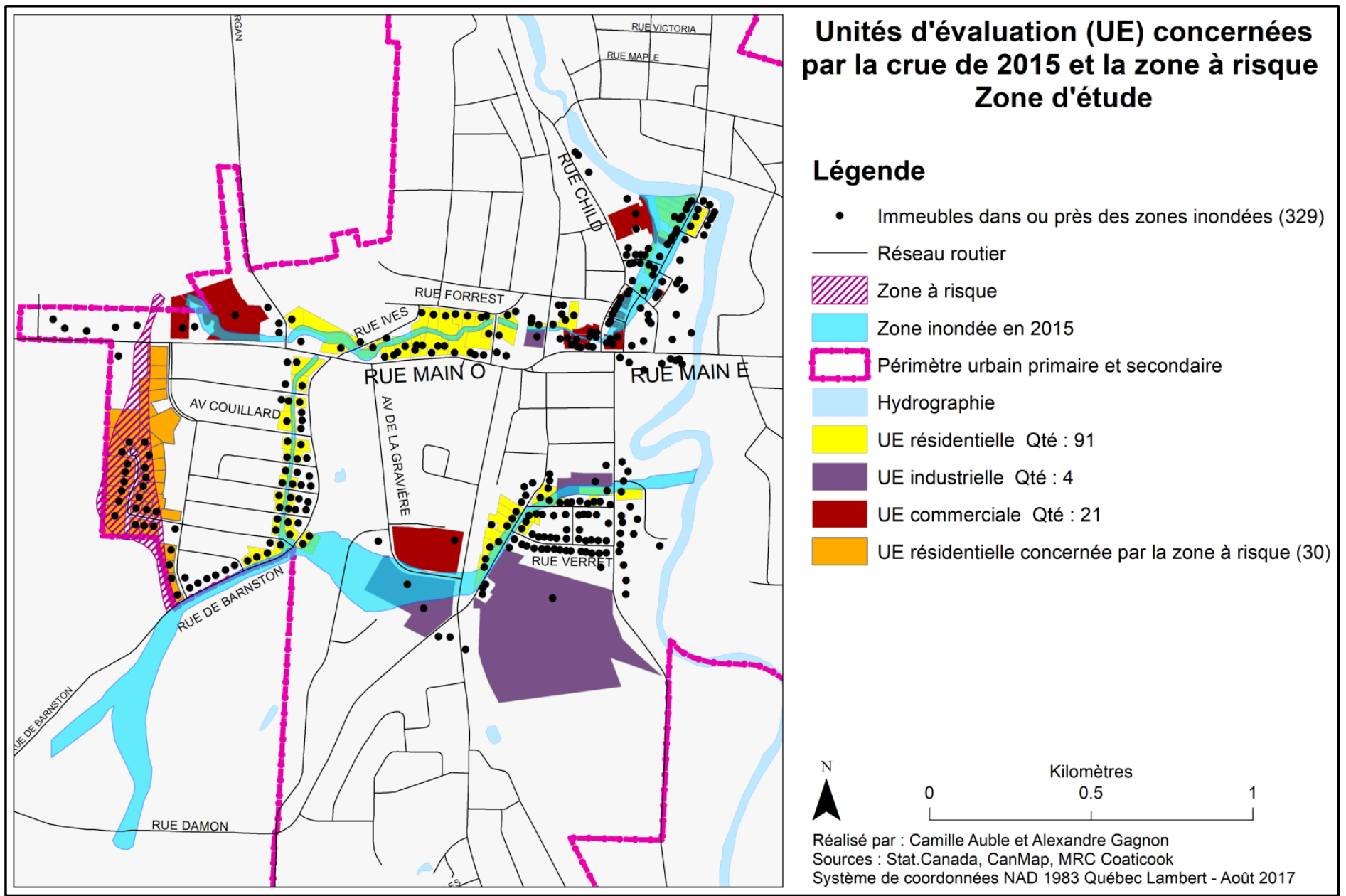

Figure 2. Unités d'évaluation (UE) concernées par la crue de 2015 et la zone à risque. Zone d'étude. Auteurs : C. Auble et A. Gagnon, 2017. Note : Le tracé de la zone inondée en 2015 a été fourni par la MRC de Coaticook (délimitation basée sur des observations terrain).

Dans la zone d'étude, on trouve le ruisseau Pratt qui trouve sa source dans des affluents agricoles à l'ouest de la ville et qui la traverse pour se jeter ensuite dans la rivière Coaticook. Le ruisseau Pratt passe près du centre-ville commercial, où il est enserré par des murs (voir figure 3) sur une bonne partie de sa longueur et est traversé par plusieurs petits ponts. Du 9 au 10 juin 2015, à la suite de pluies torrentielles, le ruisseau Pratt a débordé (voir zone inondée dans la figure 2) violemment et de manière rapide causant des dommages importants.

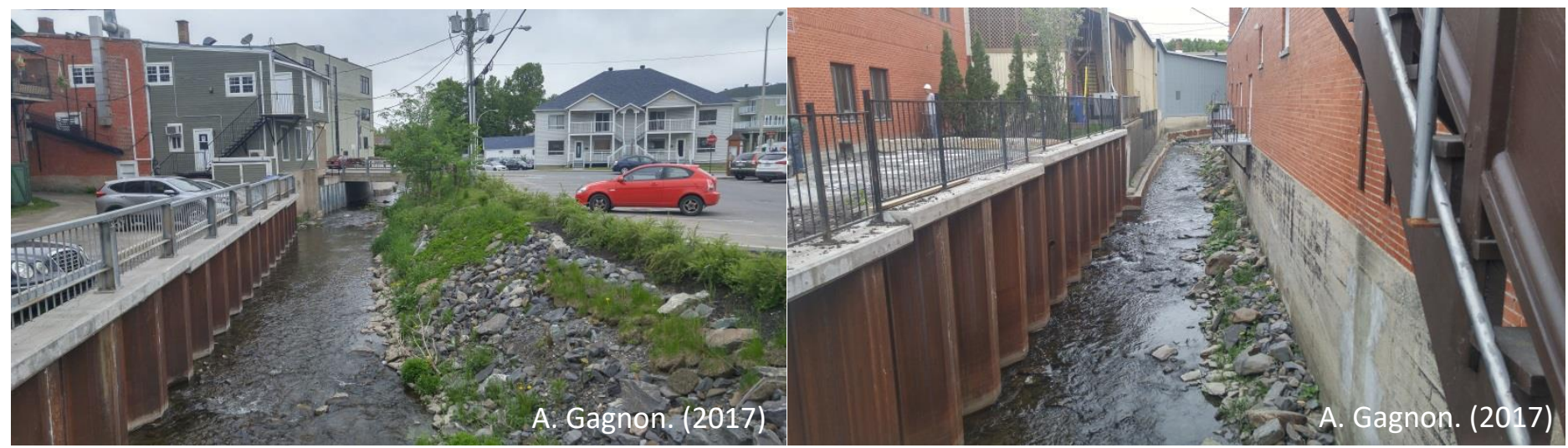

Figure 3. Vues du ruisseau Pratt au centre-ville de Coaticook. Auteur : A. Gagnon, 2017 


\section{Fondements théoriques de l'analyse de vulnérabilité}

La démarche de recherche du GECAR visant à évaluer la vulnérabilité et progresser vers l'adaptation et la résilience s'appuie notamment sur le Cadre d'action de Sendai pour la réduction des risques de catastrophe 2015-2030 (UNISDR, 2015) qui établit comme priorité la compréhension des risques de catastrophe, ce qui passe notamment par la collecte, l'analyse et la gestion de données utiles, ainsi que l'évaluation de la vulnérabilité. La recherche s'appuie également sur la Politique québécoise de sécurité civile 2014-2034 (MSP, 2014) qui reconnaît l'importance d'une approche méthodologique commune pour faciliter les échanges et la concertation entre les acteurs.

Notion fondamentale, la résilience est au cœur de la démarche de recherche. Concept complexe et évolutif, la résilience peut être décrite comme « la capacité interne dont est doté un système pour agir dans un environnement turbulent et incertain » (Thomas et Da Cunha, 2017). Son mode d'action principal est l'adaptation, visant non pas seulement à faire face et à résister aux impacts futurs, mais également à transformer de manière intentionnelle les villes et communautés afin de réduire la vulnérabilité des systèmes socioécologiques (Thomas et Da Cunha, 2017).

Un concept important est celui du risque, qui « résulte de l'interaction entre un aléa potentiel et la vulnérabilité du milieu exposé à son égard»(Morin, 2008a). Dans un cadre temporel et spatial, la gestion des risques s'articule autour de quatre phases : la prévention, la préparation, l'intervention et le rétablissement (Morin, 2008b). La prévention peut se traduire par une politique d'aménagement du territoire cherchant à diminuer les impacts potentiels d'une catastrophe. La préparation correspond à la mise en place des moyens nécessaires pour faire face aux conséquences d'un aléa. La phase d'intervention se situe lors de la crise et marque la mobilisation des divers outils, plans et services d'urgence. Finalement, le rétablissement représente la phase de reconstruction et de reprise du cours normal des activités. Durant cette phase de rétablissement, il est souhaitable d'aller au-delà du retour à la normale et de viser plutôt l'adaptation et l'augmentation de la résilience. Le retour d'expérience représente aussi une composante importante du rétablissement.

La notion d'aléa quant à elle est généralement acceptée comme identifiant un phénomène d'origine humaine (ex. : fuite industrielle de produits chimiques) ou naturelle (ex. : inondation) représentant un danger potentiel pour une société, selon un territoire et une période donnée (Pigeon, 2002). Dans le cas de l'aléa naturel, la nature et l'importance des conséquences dépendront de divers facteurs, soit «[le] champ d'action (espace), [la] magnitude (volume), [l']intensité ou [le] débit, [la] violence (impact) et [la] récurrence (fréquence) » (Thouret et D’Ercole, 1996).

Ensuite, le MSP considère que la vulnérabilité est « une condition résultant de facteurs physiques, sociaux, économiques ou environnementaux, qui prédispose les éléments exposés à la manifestation d'un aléa à subir des préjudices ou des dommages » (Morin, 2008a). Cette définition est adaptée de celle de la Stratégie internationale des Nations Unies de prévention des catastrophes. La vulnérabilité, en bref, résulte de la combinaison entre l'exposition et la sensibilité. Ainsi, les éléments exposés à un aléa sont des éléments d'un milieu susceptibles d'être affectés par un aléa et subir des préjudices ou dommages et peuvent à la fois être matériels (ex. : personnes, bâtiments, écosystèmes, etc.) et immatériels (ex. : cohésion sociale, attractivité, sentiment de sécurité, etc.) (Morin, 2008a).

La méthode d'analyse de la vulnérabilité du GECAR permet d'évaluer et de révéler la sensibilité. Cette notion de sensibilité est définie comme «la proportion dans laquelle un élément exposé, une collectivité ou une organisation, est susceptible d'être affecté par la manifestation d'un aléa » (Morin, 2008a). D'après le MSP, c'est la sensibilité qui prime sur tous les autres aspects pour établir la vulnérabilité (Morin, 2008a). Dans une optique d'adaptation, la connaissance de la sensibilité du territoire (éléments physiques) et des communautés (la population) est capitale, car la réduction de la sensibilité des éléments exposés constitue, dans bien des situations, le seul véritable moyen de réduire la vulnérabilité et même le risque (Morin, 2008a). 
4. L'analyse de la vulnérabilité : présentation des quatre indices thématiques et aspects méthodologiques

Quatre thématiques forment l'analyse de la vulnérabilité : la sensibilité sociale, la sensibilité territoriale, la capacité d'adaptation et l'accessibilité aux infrastructures en situation d'urgence (à partir de la caserne de pompiers). Il s'agit de composantes clés dans la résilience aux catastrophes : la population, le bâti et les réseaux. Chaque thématique est représentée par un indice thématique qui présente ses propres composantes (sauf l'indice d'accessibilité) et indicateurs. La combinaison des indices thématiques offre comme résultat un indice agrégé qui permet d'établir un diagnostic complet et rigoureux de la vulnérabilité. Dans la construction de l'indice agrégé, chaque indice thématique est pondéré afin d'avoir un poids égal.

Le résultat de l'évaluation de la vulnérabilité est un portrait multi échelles, robuste, complet et pouvant être à la fois décomposable ou synthétique au besoin. La figure 4 présente la structure globale de la méthode d'analyse de la vulnérabilité.

Vulnérabilité totale avec l'accessibilité aux infrastructures en situation d'urgence

Indice agrégé

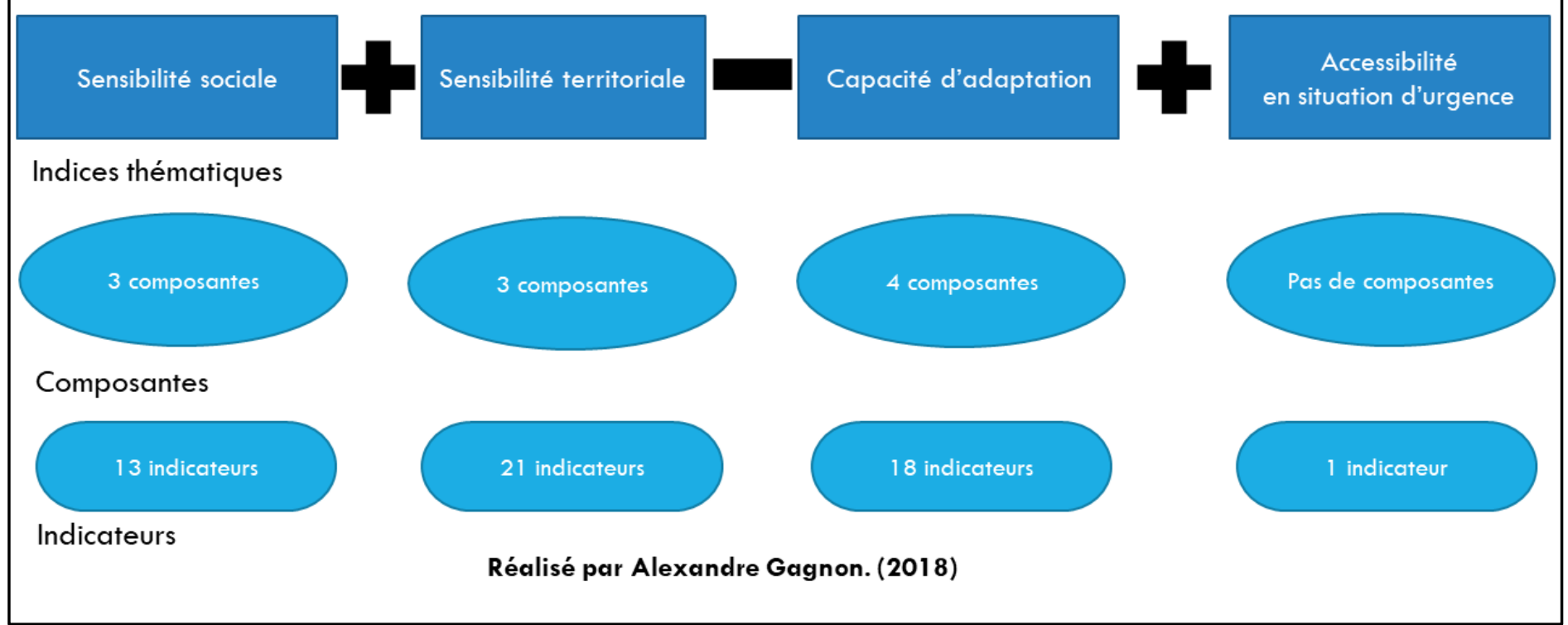

Figure 4. Structure hiérarchique de la méthode d'analyse de la vulnérabilité. Auteur : A. Gagnon, 2018 


\section{- Sensibilité sociale}

Le premier indice thématique est celui de la sensibilité sociale. Celle-ci correspond à la composition de la population faisant face à une catastrophe, composition qui influence grandement la vulnérabilité d'une municipalité (Cutter, Boruff et Shirley, 2003). Afin d'étudier cette sensibilité sociale, divers indicateurs socio-économiques et caractéristiques des individus, ménages et familles ont été séparés en composantes selon les données disponibles provenant du recensement canadien 2011 et de l'Enquête nationale auprès des ménages (ENM) 2011. La classification des indicateurs selon leur effet positif ou négatif sur la sensibilité a été évalué selon la revue de littérature réalisée par Cutter, Boruff et Shirley (2003) avec des ajustements mineurs pour le contexte du Québec. Ainsi, s'il est accepté d'après la revue de littérature que la pauvreté augmente la sensibilité de la population, certains indicateurs tels que l'ethnicité n'ont pas été utilisés car peu pertinents dans une ville comme Coaticook. Les trois composantes ayant émergé de l'analyse ont été nommées diplomation/composition familiale et âge, densité et taux d'effort et dépendance économique. La méthode mathématique utilisée afin d'identifier ces composantes est l'analyse en composantes principales (ACP). L'ACP a été popularisée dans le domaine d'évaluation de la vulnérabilité par Cutter, Boruff et Shirley (2003) qui se sont servis de cette analyse afin de concevoir un indice de vulnérabilité sociale (SoVI) aux États-Unis. Plusieurs autres chercheurs ont utilisé la méthode dans différents contextes et à plusieurs échelles. Ainsi, Thomas et Bleau (2012) ont réalisé une étude de vulnérabilité de la rive sud de la Rivière-des-Prairies sur l'île de Montréal, Guillard-Gonçalves et coll. (2015) l'ont appliqué pour caractériser et cartographier la vulnérabilité sociale du Grand Lisbonne en Espagne et Tate, Cutter et Berry (2010) s'en sont servis pour construire un indice de vulnérabilité sociale à l'échelle du comté de Charleston, en Caroline du Sud.

\section{- Sensibilité territoriale}

La sensibilité territoriale correspond quant à elle aux infrastructures «localisables susceptibles d'engendrer et de diffuser leur vulnérabilité à l'ensemble du territoire, provoquant des effets qui peuvent perturber, compromettre, voire interrompre son fonctionnement et son développement » (D'Ercole et Metzger, 2009). Ainsi, dans l'optique de présenter un portrait de la vulnérabilité d'une société face aux risques de catastrophe, il est nécessaire d'évaluer l'organisation du territoire, son aménagement ainsi que les ressources de la gestion de crise. L'identification des lieux où se concentre la sensibilité permet de mieux comprendre le territoire, de repérer ses faiblesses dans une optique de prévention et d'adaptation, de le préparer judicieusement à toute éventualité et d'y intervenir en connaissance de cause en cas d'urgence. La méthode utilisée par le GECAR consiste en un comptage des infrastructures par unité de maillage géolocalisée, selon différents types d'infrastructures puis de manière agrégée par composantes. Cette méthode s'appuie sur les travaux de Thomas et Bleau (2012) et sur les travaux de Thomas et coll. (2017) qui ont affiné les analyses notamment dans le cas de la ville de Saint-Raymond. Les données utilisées sont fournies par la MRC et majoritairement extraites du rôle d'évaluation foncière. Le rôle d'évaluation foncière est un inventaire des immeubles situés sur le territoire d'une municipalité, évalués sur une même base et à une même date (MAMOT, 2014). Il contient généralement une donnée sur l'utilisation prédominante de l'immeuble et c'est cette donnée qui a été extraite dans le cadre de l'étude. L'indice a été divisé en trois composantes : les logements sensibles, les infrastructures critiques et les infrastructures d'intérêt.

\section{- Capacité d'adaptation}

Ensuite, la capacité d'adaptation correspond à la faculté des populations ou des systèmes naturels de diminuer les impacts négatifs potentiels d'un événement donné ou d'en augmenter les impacts bénéfiques, en prenant des mesures appropriées (Thomas et Da Cunha, 2017). La capacité d'adaptation a été divisée en quatre composantes : le capital social, le développement économique, les compétences communautaires et la condition des infrastructures. Dans le cas de la présente recherche, des indicateurs disponibles provenant majoritairement du recensement canadien 2011 et de 1'ENM 2011 ont été utilisés afin de brosser un portrait des composantes. D'autres approches plus extensives en 
termes de temps et de ressources pourraient privilégier des questionnaires et visites terrain. La notion de capital social découle de l'idée que les individus utilisent des ressources liées aux réseaux sociaux afin d'en tirer des bénéfices (Norris et coll., 2008). Le capital social se manifeste dans les structures publiques, les liens des réseaux informels, ainsi que dans le support social, c'est-à-dire les interactions sociales qui fournissent de l'aide aux individus et qui les incorporent dans une panoplie de relations sociales considérées comme étant disponibles en temps de crise (Barrera, 1986, cité dans Norris et coll., 2008). Le développement économique se mesure par rapport à la diversité, l'équité et au volume de ressources disponibles. À ce sujet, des études ont démontré que des personnes avec un statut socioéconomique faible connaissaient des conséquences psychologiques plus importantes que celles ayant un profil socio-économique élevé après une catastrophe (Norris et coll., 2002 cité dans Norris et coll., 2008). Les compétences communautaires se rattachent à la capacité des membres d'une communauté à collaborer efficacement afin d'identifier les problèmes et les pistes de solutions possibles. En général, on considère une communauté compétente dès lors qu'elle possède les aptitudes nécessaires à la prise de décision, à la délibération, à la collecte d'information et à l'atteinte de consensus (Cottrell, 1976, cité dans Norris et coll., 2008). Enfin, la notion de capacité d'adaptation des infrastructures représente la capacité du cadre bâti à faire face à l'aléa et à être capable de résister sur le court terme et de s'adapter de manière planifiée sur le long terme.

- Accessibilité aux infrastructures en situation d'urgence (à partir de la caserne de pompiers)

Finalement, l'accessibilité aux infrastructures en situation d'urgence correspond à la capacité à atteindre un point précis du territoire en un temps donné lors d'une inondation. Dans le cas de la présente recherche, le temps de parcours est mesuré à partir de la caserne de pompiers. L'accessibilité (évacuation des zones sinistrées, accès des secours) est à prendre en compte lors de la création d'un indice reflétant la sensibilité totale d'une ville (Demoraes, 2009). Les données utilisées proviennent d'Adresses Québec et du rôle d'évaluation foncière. Lors de la phase d'intervention (en cas d'urgence), l'accessibilité aux logements notamment est primordiale. L'impossibilité de se rendre à l'hôpital ou pour les pompiers de rejoindre une habitation ou une entreprise manipulant des produits dangereux peut avoir des conséquences dramatiques. Ceci affecte directement la sensibilité générale d'un système urbain. C'est dans cette optique qu'il est essentiel de connaître les zones potentiellement sujettes à être complètement isolées afin d'anticiper leurs besoins et améliorer la redondance et l'accès au réseau routier y compris les ponts. L'établissement de scénarios particuliers et l'analyse d'impact de la perte de segments routiers clés à l'aide d'isochrones de temps de parcours s'inscrivent dans cette optique d'anticipation. Cette méthode s'appuie sur les travaux de Thomas et coll. en 2017 et de Serrhini (dir.) (2009). 


\subsection{L’analyse de la vulnérabilité : résultat de l'évaluation}

La section suivante comprend les résumés des résultats à Coaticook pour chacun des indices thématiques et pour l'indice agrégé de la vulnérabilité totale avec l'accessibilité aux infrastructures en situation d'urgence.

\section{- Sensibilité sociale}

Sur le plan de la sensibilité sociale, la zone concernée par les inondations du ruisseau Pratt en 2015 est particulièrement sensible : les quatre aires de diffusion avec une très forte sensibilité s'y retrouvent (voir figure 5). On trouve dans ces aires de diffusion, de manière générale et selon les données disponibles, peu de gens possédant des diplômes universitaires (en deçà de $10 \%$ ), beaucoup de ménages avec une seule personne (environ $35 \%$ ) et aussi beaucoup de personnes âgées de 65 ans et plus (environ $24 \%$ ). Il faut donc penser à renforcer la capacité à faire face et à s'adapter aux inondations en travaillant sur la sensibilisation, l'accompagnement technique, la prévention et l'évacuation en tenant compte de la réalité sociale de ces aires de diffusion et des besoins particuliers (personnes âgées, familles monoparentales, locataires, personnes moins nanties, etc.).

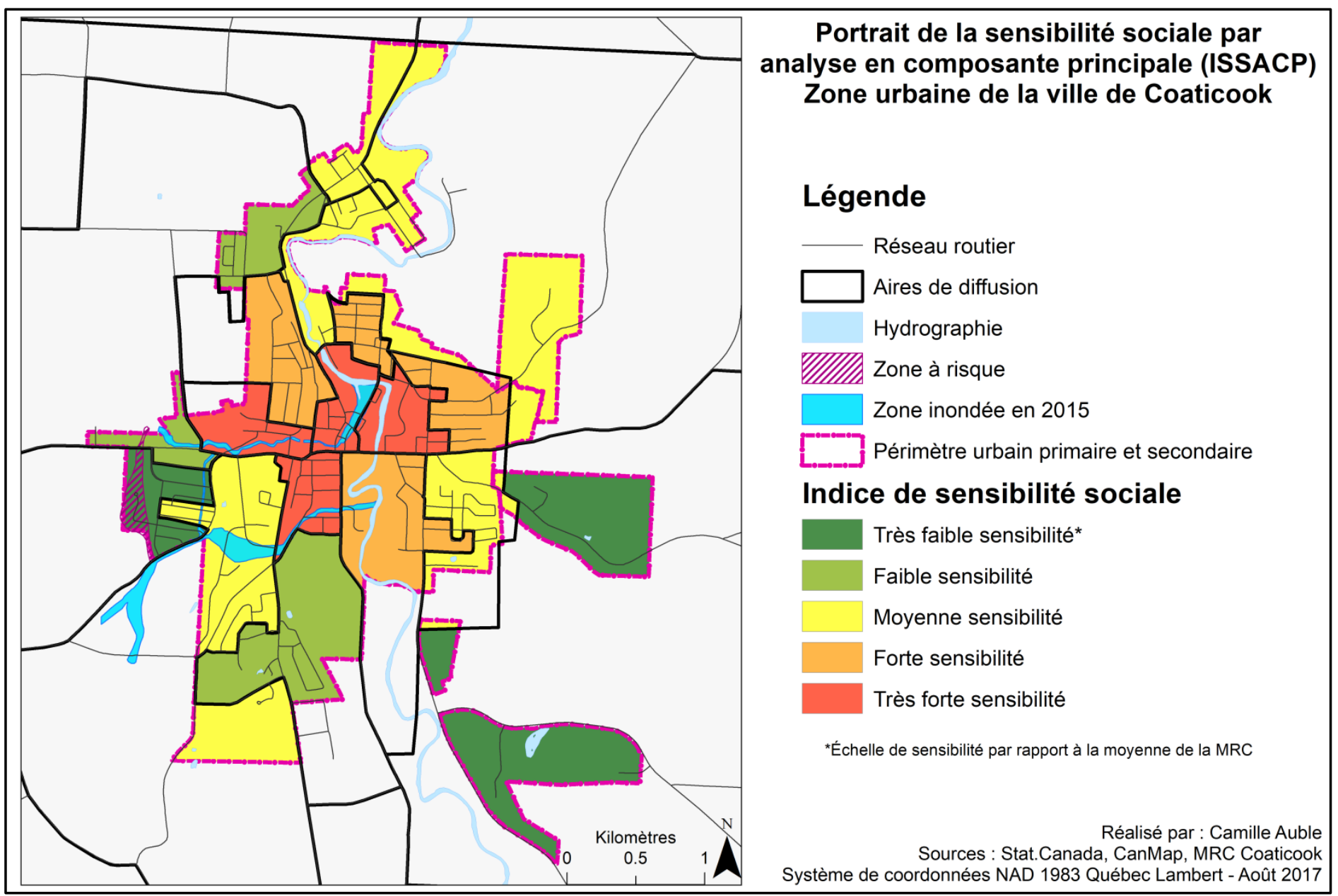

Figure 5. Portrait de la sensibilité sociale par analyse en composante principale (ISSACP).

Auteur : C. Auble, 2017 


\section{- Sensibilité territoriale}

Sur le plan de la sensibilité territoriale, en observant la carte à l'aide d'un maillage de 250 mètres $x$ 250 mètres, on constate que les zones les plus sensibles correspondent le plus souvent aux zones résidentielles denses (voir figure 6). On retrouve ces zones au centre-ville et près des rues Main Ouest et Est. La zone concernée par les inondations du ruisseau Pratt en 2015 affiche une sensibilité élevée, voire très élevée près de la rivière Coaticook, mais moins élevée vers l'ouest et le sud, lorsque l'on s'éloigne du centre-ville. Dans celui-ci, on trouve des dizaines de commerces et de logements par maille, la caserne de pompiers, l'hôtel de ville, des segments de réseau routier supérieur et du réseau d'aqueduc et une résidence pour personnes âgées. La zone commerciale de la rue Main Ouest affiche quant à elle une forte sensibilité. Le centre-ville est le cœur commercial de la ville : une mauvaise préparation, intervention et rétablissement/adaptation dans ce secteur clé pourrait entraîner d'importantes conséquences économiques (enjeu de continuité des affaires) et affecter un grand nombre d'employés et d'employeurs dans leur vie quotidienne en fragilisant leur capacité à faire face à une nouvelle catastrophe. Il est donc indispensable de conscientiser les habitants et commerçants concernant leur vulnérabilité.

Portrait de la sensibilité territoriale (IST)
avec un maillage de 250m par 250m
Toutes les infrastructures
Zone urbaine de la ville de Coaticook

Figure 6. Portrait de l'indice de sensibilité territoriale (IST) (maillage de $250 \mathrm{~m}$ par $250 \mathrm{~m}$ ). Auteur: A. Gagnon, 2017. 
- Capacité d'adaptation

Au niveau de la capacité d'adaptation, on observe que celle-ci est plus faible au centre-ville à la jonction des rues Main Ouest et Est et dans les aires de diffusion adjacentes (voir figure 7). Les aires de diffusion avec une capacité d'adaptation élevée sont toutes situées aux extrémités du périmètre urbain. De manière générale, on peut affirmer que la zone concernée par les inondations du ruisseau Pratt possède une capacité d'adaptation faible à très faible, exceptée dans sa partie sud-ouest. Ce portrait met en lumière la très forte vulnérabilité liée à la capacité d'adaptation des zones du centre-ville. Ainsi, de manière générale, cette zone compte une population majoritairement locataire, un faible pourcentage de la population gagnant au-delà de 30000 \$ et environ la moitié de la population qui a déménagé dans les cinq dernières années. Que ce soit au niveau de la prévention, de la sensibilisation ou de l'aménagement du territoire, des actions doivent être menées afin d'améliorer significativement la résilience du centre-ville commercial et faire augmenter sa capacité d'adaptation.

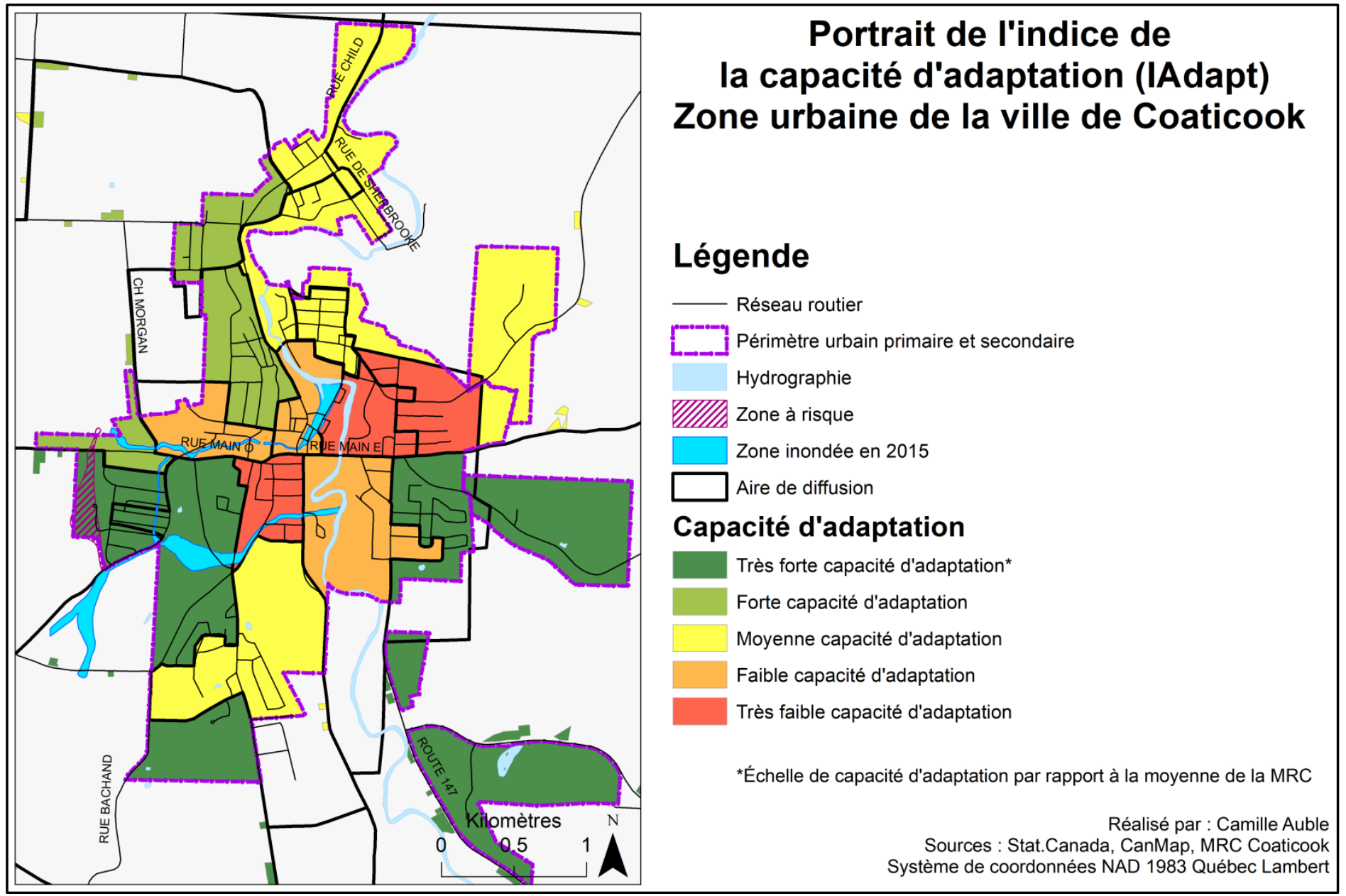

Figure 7. Portrait de l'indice de la capacité d'adaptation (IAdapt). Auteur : C. Auble, 2017. 
- Accessibilité aux infrastructures en situation d'urgence (à partir de la caserne de pompiers)

La figure 8 ci-dessous constitue le portrait de l'accessibilité aux infrastructures au départ de la caserne de pompiers en situation d'urgence (en cas d'inondation). Elle a été composée grâce à la méthode d'analyse de la vulnérabilité et améliorée grâce aux informations fournies par monsieur Simon Morin, responsable des services extérieurs, sur les routes coupées durant l'inondation de juin 2015. Les zones inaccessibles y sont illustrées en blanc et les routes coupées sont représentées par les traits gris. Lorsque certains segments de routes ne sont plus praticables, l'accessibilité à certaines infrastructures est lourdement impactée du fait que celles-ci sont dépendantes du réseau routier. La zone inaccessible la plus préoccupante au niveau de la vulnérabilité est la zone située près de la rue Wellington (route 147), bloquant potentiellement une partie de l'accès vers la rue Main Ouest et le pont de la rue Saint-Jean-Baptiste. La zone à risque est préoccupante, car située plus loin à l'ouest et aussi par le fait qu'elle contient une seule voie d'accès se terminant en cul-de-sac, la Rue des Fougères. On note également l'accès difficile à l'école primaire Sacré-Cœur et à la résidence pour personnes âgées Le Bel Âge qui comprend 22 unités de chambres simples et est aménagée de façon à pouvoir accueillir des personnes à mobilité réduite. Selon les caractéristiques de l'inondation, certaines routes peuvent être impraticables même si elles ne l'ont jamais été auparavant. Lorsque ces routes occupent des rôles stratégiques dans le réseau routier, les conséquences sur l'accessibilité effective peuvent être majeures. Ainsi, on peut penser au pont de la rue Main Est : s'il était bloqué, il serait bien plus difficile de rejoindre la partie est de la ville. La rue Main Ouest aussi de manière générale est un axe prioritaire sensible et permet de rejoindre le développement résidentiel à l'ouest lorsque la rue Ives est impraticable. Cette cartographie aide à anticiper les enjeux d'accessibilité et à préparer les services concernés et la population.

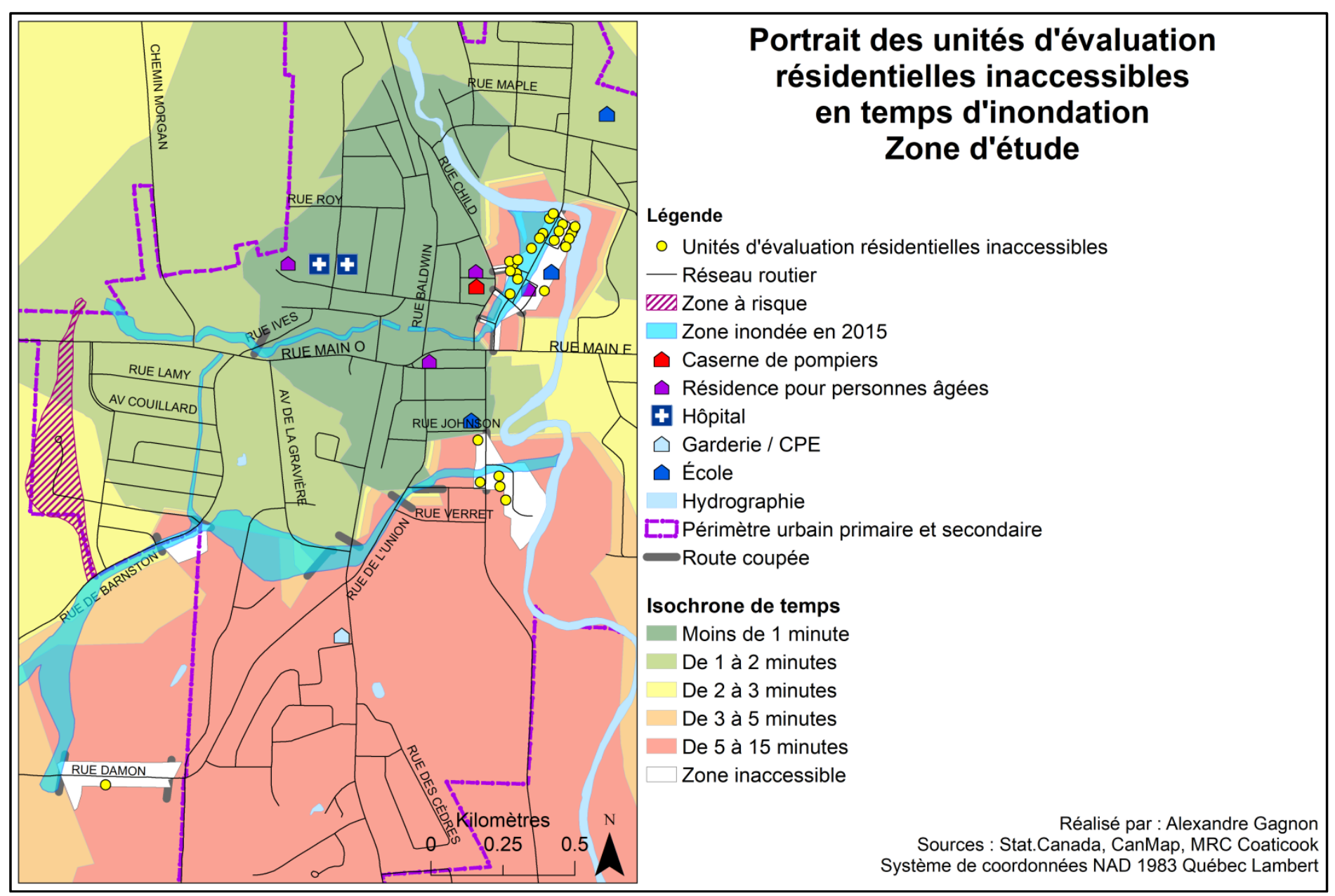

Figure 8. Portrait de l'accessibilité en situation d'urgence au départ de la caserne de pompiers incluant les unités d'évaluation inaccessibles. Auteur : A. Gagnon, 2018. 
- Vulnérabilité totale avec l'accessibilité aux infrastructures en situation d'urgence (à partir de la caserne de pompiers)

De manière globale, les résultats de l'analyse de la vulnérabilité totale avec l'accessibilité aux infrastructures en situation d'urgence (voir figure 9) confirment la vulnérabilité du centre-ville, qui en plus d'être une zone exposée aux inondations, compte des populations sensibles (âgées, moins nanties, locataires, etc.) en grande proportion et avec une capacité d'adaptation faible à très faible, un nombre élevé d'infrastructures et particulièrement de commerces et devra prendre en compte les nombreux enjeux d'accessibilité réels et potentiels dans l'évacuation et le rétablissement. Un renforcement des connaissances, des capacités des groupes communautaires et des stratégies d'aménagement du territoire et d'intervention est nécessaire. Il faudra faire preuve de précaution et prendre en compte les éléments ajoutant de l'incertitude dans les mesures de prévention: par exemple les changements climatiques, la possibilité de subir un embâcle, l'espace de liberté de la rivière Coaticook, les potentiels effets dominos, etc. L'immunisation et/ou la protection des immeubles situés dans ou près des zones inondées peut être considérée, tout comme l'effet du potentiel bassin de rétention et la création ou mise en valeur d'infrastructures naturelles. Plusieurs stratégies d'adaptation complémentaires sont nécessaires afin d'assurer le renforcement de la résilience de la ville et de la communauté.

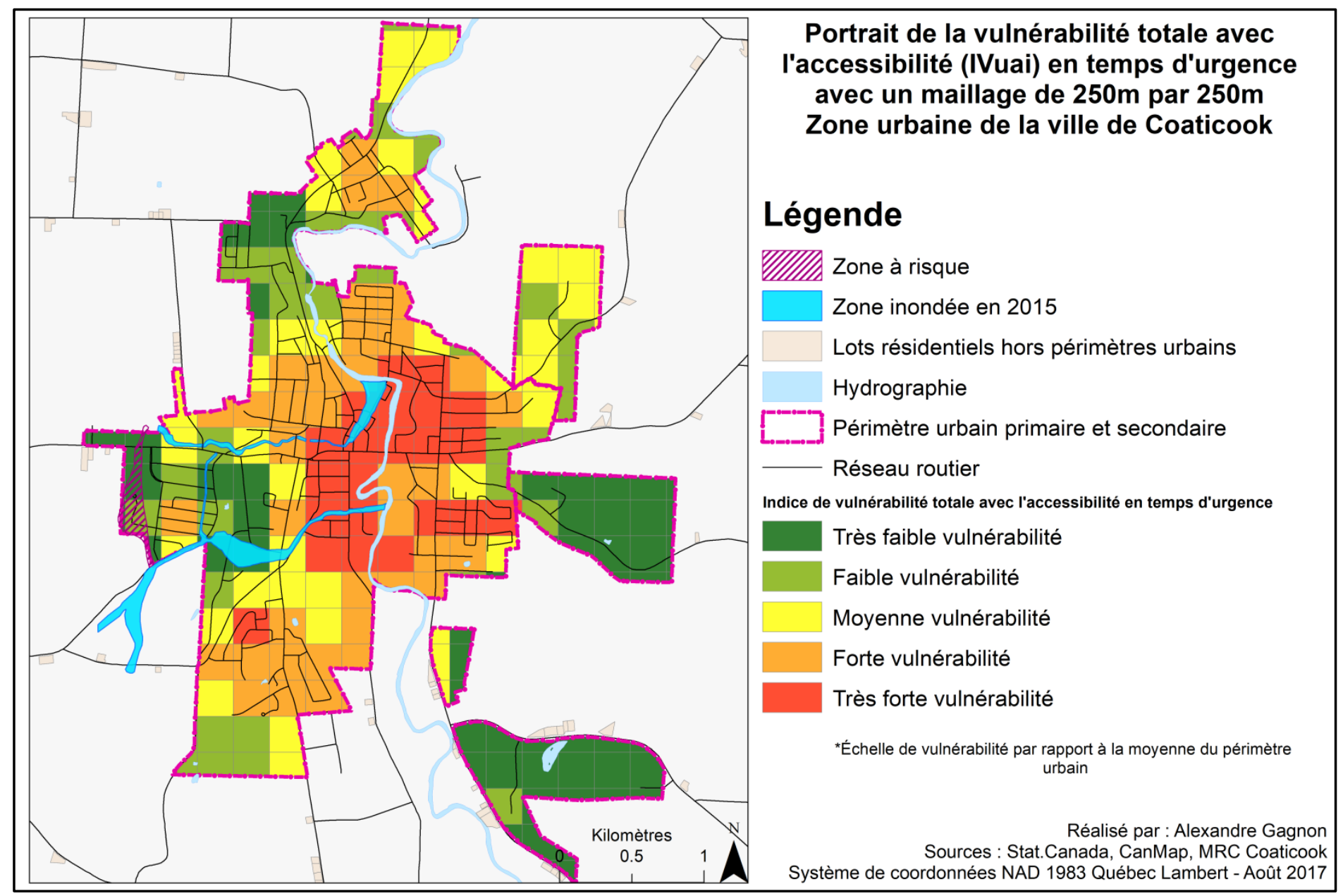

Figure 9. Portrait de l'indice de vulnérabilité totale avec l'accessibilité aux infrastructures en situation d'urgence (à partir de la caserne de pompiers) Auteur : A. Gagnon, 2017. 
5. L'analyse des dommages : dépenses conséquentes dans la zone d'étude, particulièrement au centre-ville

En qui concerne l'analyse des dommages, les données d'aide financière du MSP associées aux demandes de remboursement post-inondation ont permis de mettre en lumière les sommes considérables qui ont été consenties à la municipalité (2 081105 \$), aux particuliers (808 982 \$) et aux entreprises (802 315 \$) entre 1998 et 2015, pour un total de 3692402 \$. Tous les montants sont en dollars de 2017. Pour les inondations de juin 2015 plus précisément, le portrait d'endommagement a permis de relever le montant important accordé à la municipalité (1 200000 \$) utilisé majoritairement pour la reconstruction de murs encadrant le ruisseau près de la rue Child. Chez les particuliers, les allocations de départ et frais de démolition pour deux résidences, les réparations majeures et les travaux d'urgence ont constitué $96 \%$ de la facture totale qui s'élève à $579971 \$$. Au niveau des entreprises, le montant validé s'est établi à 170495 \$. Sur le plan spatial, le portrait d'endommagement a permis de confirmer que la plus grande partie des dommages a été concentrée au centre-ville. Le secteur résidentiel à l'ouest a également été touché de manière significative, contrairement au secteur de la rue d'Ottawa (au sud du centre-ville), qui n'a pas été endommagé malgré qu'il ait été touché par l'inondation (voir figure 10). En faisant une corrélation avec l'analyse de la vulnérabilité, il a été possible de constater que la plus grande part des montants réclamés se trouvent tous dans des zones très vulnérables, surtout concentrés dans le secteur où la zone inondée en 2015 a rejoint la rivière Coaticook. Le développement résidentiel à l'ouest présente une vulnérabilité généralement faible, mais des montants conséquents y ont été réclamés.

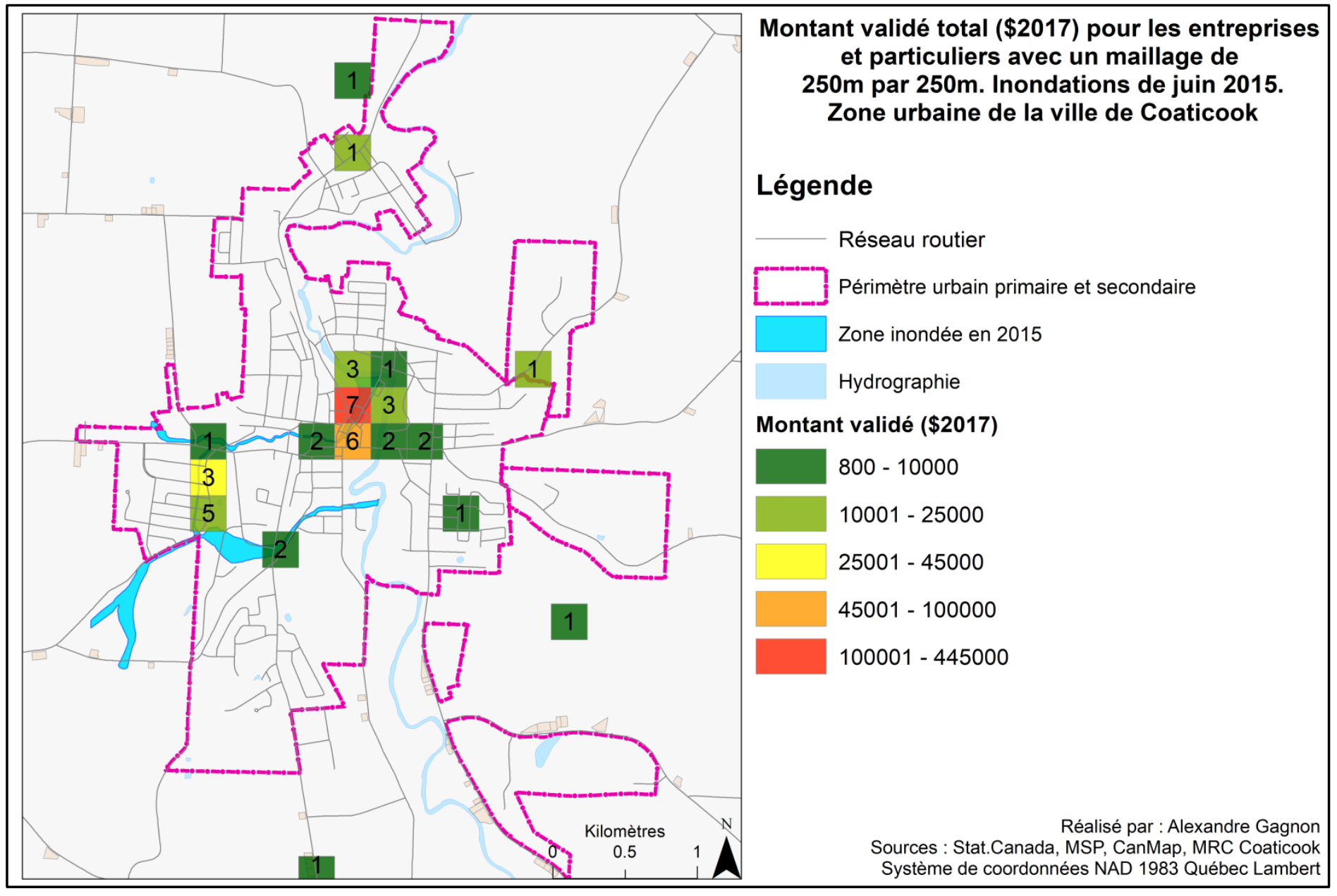

Figure 10. Montant validé total pour les entreprises et particuliers avec un maillage de $250 \mathrm{~m}$ par $250 \mathrm{~m}$. Inondations de juin 2015. Zone urbaine de la ville de Coaticook. Auteur : A. Gagnon, 2017. 
6. Impliquer les acteurs locaux et les citoyens dans la méthode d'analyse de la vulnérabilité : la plus-value de la connaissance locale pour l'adaptation

L'implication des acteurs locaux et des citoyens permet à chacun de partager sa vision du risque et des enjeux vulnérables à Coaticook et oriente les actions et stratégies à venir. Cette implication et collaboration active a débuté dès le démarrage du projet. En outre, l'implication des acteurs locaux s'est traduite par 2 séances de présentation et de discussion de résultats (14 novembre 2017 et 16 juillet 2018), une table participative (16 juillet 2018) et un sondage auprès des citoyens (été 2018) sur la perception du risque et les mesures d'adaptation. Ces échanges ont permis d'apporter un éclairage précieux sur les besoins tant en termes de connaissance du risque que de mesures d'adaptation.

\subsection{Séance de présentation et de discussion de résultats et table participative : cartographie des enjeux et solutions nouvelles}

La séance de présentation et de discussion du 16 juillet 2018 a permis aux acteurs locaux de se rencontrer tous autour de la même table et de partager des informations précises qui ne sont pas normalement partagées. De plus, il a été possible de discuter de différentes stratégies d'adaptation aux effets multiples et de mettre à jour les acteurs et l'équipe de recherche sur les projets qui sont en cours ou qui viennent tout juste de débuter. La séance a été organisée et structurée par les auxiliaires de recherche Alexandre Gagnon et Anna Michetti et par la professeure Isabelle Thomas. Autour de la table se trouvaient François Fréchette (directeur général de la ville de Coaticook), Simon Morin (responsable des services extérieurs) avec Erika Gilbert (employée de la ville), Jean-Pierre Pelletier (citoyen touché par l'inondation de 2015), Marie-Claude Bernard (coordonnatrice de projet en ressources naturelles de la MRC) avec Aglaé Landry-Boisvert (stagiaire), Catherine Frizzle (coordonnatrice de projets au COGESAF) et Catherine Otis (conseillère en sécurité civile du MSP). Les expertises de chacun et leur échelle d'intervention étaient particulièrement complémentaires.

Grace à cette séance de co-construction, chacun est reparti avec une compréhension fortement améliorée du contexte et de l'avancement de la ville dans le dossier des inondations. Les manières d'aborder le risque ont pu évoluer grâce au projet de recherche en permettant aux acteurs de considérer que la meilleure adaptation résulte souvent d'un cocktail de solutions et de mesures sans regret concernant la prévention, préparation, l'urgence et le rétablissement au-delà de la situation normale. Il a également été souligné qu'il fallait considérer chaque action posée non pas seulement comme une action permettant de réduire les risques d'inondations, mais aussi comme une action visant l'amélioration de la qualité de vie des citoyens. Les acteurs ont notamment affirmé que les cartes et l'analyse de vulnérabilité permettaient d'officialiser la connaissance et pourraient contribuer à une potentielle révision du plan de mesures d'urgence ou la mise sur pied d'un plan particulier pour le risque d'inondation.

La table participative réalisée le 16 juillet 2018 dans le cadre de la séance de présentation et de discussion de résultats a permis de faire ressortir la connaissance locale en guidant les participants dans leur réflexion par trois questions :

1. Selon vos connaissances locales du territoire de la ville de Coaticook, pouvez-vous identifier et prioriser les enjeux de vulnérabilité ?

2. Pourquoi sont-ils vulnérables ? Comment ces enjeux vulnérables affectent-ils le bon fonctionnement de la communauté en cas d'inondation?

3. Durant quelle(s) phase(s) de la gestion des risques ces enjeux présentent-ils de la vulnérabilité ? À quelle échelle?

Les acteurs locaux ont pu répondre aux questions sur une feuille préparée à l'occasion et ont pu dessiner sur une carte régionale et sur une carte locale avec des enjeux visibles et encercler les enjeux prioritaires (voir figure 11). 


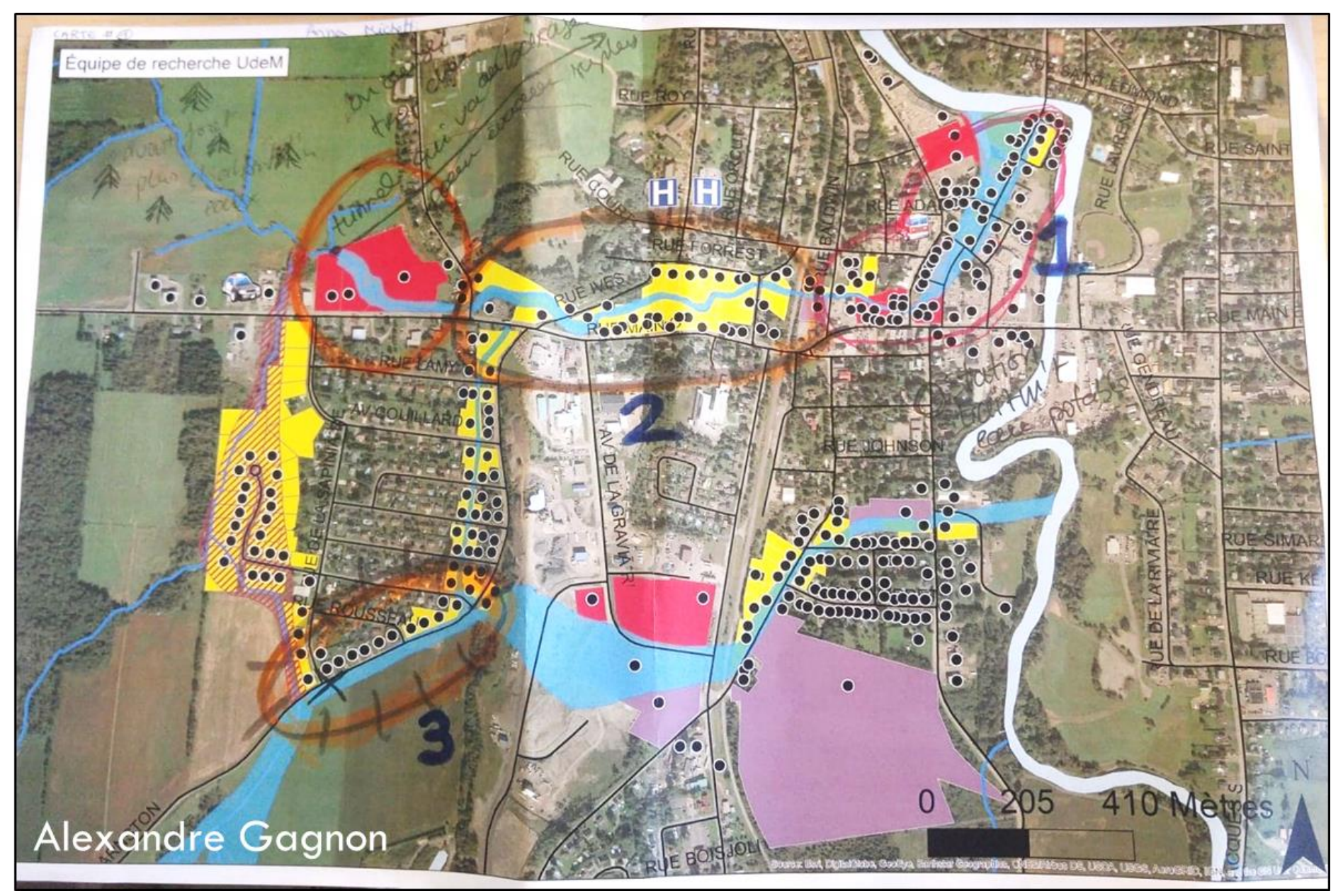

Figure 11. Zones vulnérables spatialisées par une équipe d'acteurs locaux (Erika Gilbert, François Fréchette, Catherine Frizzle, Aglaé Landry-Boisvert supervisés par Anna Michetti) durant la table participative.

De manière spatialisée, les deux équipes ont mentionné que la zone la plus vulnérable était le centreville (secteur identifié par le chiffre 1 dans la figure 11) pour plusieurs raisons. Il s'agit d'abord d'une zone où les espaces sont presque tous occupés et où plusieurs bâtiments sont déjà construits très près du ruisseau. On retrouve dans le secteur un grand nombre de commerces, particulièrement sur la rue Child et une concentration du trafic routier. La caserne de pompiers est près de la rue commerciale, mais l'évacuation est difficile en cas d'urgence à cause du nombre important de personnes à évacuer potentiellement et les nombreuses rues qui peuvent être fermées. Les inondations ont un impact important pour les commerçants, certains devant fermer boutique. Il s'agit également d'une zone très peu perméable. Une équipe a soulevé qu'une autre zone vulnérable était constituée par les secteurs résidentiels situés sur la rue Ives et la rue Main (secteurs identifiés par le chiffre 2) puisqu'ils sont difficiles à évacuer. Un autre point vulnérable mentionné est le ponceau de la rue Barnston (secteur identifié par le chiffre 3), qui n'avait pas suffi à retenir toute l'eau provenant du ruisseau Cloutier, le surplus ayant débordé, arraché une partie de la route et dévalé une pente puis allant jusqu'à rejoindre la rivière Coaticook. La ville prévoit agrandir ce ponceau afin de régler le problème. D'autres enjeux ayant été mentionnés concernent la nécessité d'avoir une vision d'ensemble et de protéger l'environnement au niveau du bassin versant. Les enjeux de défrichement et de drainage des terres agricoles qui peuvent accentuer le ruissellement ont également été soulignés.

\section{Questionnaire aux citoyens sur la perception du risque et les mesures d'adaptation : l'état de la situation et les besoins de la population}

De manière complémentaire à la méthode d'analyse de la vulnérabilité, le GECAR a également exploré la perception du risque chez les citoyens et les besoins en termes d'adaptation à l'aide d'un 
sondage au porte-à-porte aux citoyens habitant dans la zone d'étude réalisé à l'été 2018. Cet outil a permis d'aborder plusieurs questions concernant notamment les mesures prises par les citoyens pour s'adapter depuis l'inondation de 2015, leur niveau de préparation et de sensibilisation et leurs besoins en termes d'outils et de ressources. Avec 31 répondants au porte-à-porte et 4 dans une version en ligne, plusieurs dynamiques intéressantes ont pu émerger du questionnaire. Il s'agit d'une démarche que le GECAR intègre de plus en plus dans ses recherches vu l'importance d'un bon retour d'expérience pour aider les citoyens et acteurs locaux à s'adapter.

\section{De l'évaluation du risque à l'adaptation et la résilience}

La démarche de recherche et les efforts des acteurs locaux ont certainement fait progresser la compréhension du risque à Coaticook. Déjà, à l'heure actuelle, de nombreuses initiatives existent ou seront mises sur pied afin d'améliorer la gestion du risque et renforcer l'adaptation et la résilience à Coaticook.

Au niveau de la mitigation du risque, la démarche de recherche menée par l'Université Laval a permis d'évaluer et de préciser les besoins concernant un éventuel bassin de rétention. La démarche de l'Université de Montréal, notamment via l'analyse de l'accessibilité aux infrastructures, pourrait quant à elle mener à l'amélioration des pratiques et des mesures en situation d'urgence, des mesures d'adaptation et de communication du risque.

Au niveau de la planification du territoire, la MRC de Coaticook a fait preuve d'audace en adoptant un règlement (6-25.1) modifiant le SADD (Schéma d'aménagement et de développement durable) pour mettre à jour la cartographie des contraintes naturelles du bassin versant de la rivière Coaticook en intégrant les zones inondables, les zones de mobilité, les cônes alluviaux identifiés de même que les milieux humides identifiés par Canards Illimités en 2016 pour le bassin versant de Coaticook, tout en prévoyant de nouvelles dispositions sur les cônes alluviaux et à l'intérieur de la zone de mobilité de la rivière Coaticook (MRC de Coaticook, 2018). Une zone inondable de grand courant a notamment été identifiée et cartographiée pour le ruisseau Pratt.

À l'échelle de la ville, des discussions avec les acteurs locaux ont fait ressortir la potentielle révision du plan de mesures d'urgence. Il a également été suggéré de mettre sur pied un plan de sécurité civile consacré entièrement au risque inondation. François Fréchette, directeur général de la ville, a d'ailleurs mentionné que l'analyse de la vulnérabilité réalisée par le GECAR pourrait permettre « d'officialiser la connaissance » et de «donner du poids par des cartes et des chiffres » à des mesures et arguments par exemple d'un potentiel plan de mesures d'urgence révisé. Selon lui, ce travail de recherche pourrait permettre de représenter sur une carte ce que certains employés et résidents perçoivent plutôt instinctivement afin de mieux le visualiser et pouvoir faire ressortir plus clairement les enjeux.

Au niveau de la sensibilisation et de la communication du risque, les discussions avec les acteurs locaux ont de confirmer l'importance d'un système d'alerte et d'information pour les inondations. L'importance d'utiliser plusieurs médias ciblés afin de rejoindre efficacement la population a été mise de l'avant. La mise sur pied d'un comité citoyen est également étudiée.

\section{Conclusion : s'adapter au-delà du retour à la normale}

S'appuyant sur les connaissances et travaux déjà développés par d'autres chercheurs et instances publiques, la méthode développée par le GECAR se démarque en proposant un outil permettant une analyse multi échelles, adapté au contexte québécois notamment au niveau des données disponibles et suffisamment souple pour être utilisé au sein de diverses municipalités. Les analyses permettent de dresser des diagnostics sur les réalités locales et territoriales et donc de développer des stratégies d'adaptation et de prévention répondant aux besoins locaux. Ainsi, les résultats portant sur la 
municipalité de Coaticook démontrent sa capacité à faire ressortir les enjeux sociaux, territoriaux, liés à la capacité d'adaptation et à l'accessibilité des infrastructures. Elle permet d'avoir un portrait complet, robuste, décomposable, mais aussi synthétique de la vulnérabilité. La démarche de recherche combinant une analyse scientifique quantitative et qualitative poussée tout en assurant un suivi et une implication des acteurs locaux, par l'entremise d'un processus participatif et itératif, a permis de faire avancer la connaissance du risque et a mis en relation des acteurs clés. Ce processus de coproduction de la connaissance et de travail en équipe est un atout indéniable de la méthode qui renforce la résilience locale.

La démarche de résilience et d'adaptation peut se poursuivre désormais sur de bonnes bases en considérant notamment des ajustements au cadre d'aménagement du territoire, la poursuite de l'analyse du projet de bassin de rétention et la bonification de la planification et de la gestion du risque qui peut passer par un plan de sécurité civile propre à la gestion des inondations, particulièrement au centreville. Également, il est primordial de favoriser l'implication des acteurs locaux et des citoyens à de multiples échelles et l'élaboration d'un système de sensibilisation, d'information et d'accompagnement auprès des particuliers afin qu'ils puissent être prêts et qu'ils puissent s'adapter convenablement en tenant compte de l'incertitude et du potentiel destructeur des inondations à Coaticook. Enfin, des stratégies ciblées en termes de planification du territoire, de mitigation et d'adaptation permettront de construire sur le long terme un territoire et des collectivités résilientes. Un changement de paradigme est nécessaire : la ville de Coaticook doit désormais s'adapter en allant au-delà du retour à la normale afin de devenir plus résiliente.

\section{Bibliographie}

Cutter, S. L., Boruff, B. J., \& Shirley, W. L. (2003). Social vulnerability to environmental hazards. Social Science Quarterly, 84(2), 242-261. doi:10.1111/1540-6237.8402002. Repéré à https://onlinelibrary.wiley.com/doi/full/ $10.1111 / 1540-6237.8402002$

Demoraes, F. (2009). De l'intérêt d'une étude sur la vulnérabilité des réseaux routiers et de transport pour la compréhension des vulnérabilités territoriales - le cas du District métropolitain de Quito (Équateur). Cybergeo : European Journal of Geography, document 446. doi : 10.4000/cybergeo.22101. Repéré à https://journals.openedition.org/cybergeo/22101

D’Ercole, R. et Metzger, P. (2009). La vulnérabilité territoriale : une nouvelle approche des risques en milieu urbain. Cybergeo : European Journal of Geography. doi : 10.4000/cybergeo.22022. Repéré à http://cybergeo.revues.org/ 22022

Guillard-Goncalves, C., Cutter, S. L., Emrich, C. T., \& Zezere, J. L. (2015). Application of Social Vulnerability Index (SoVI) and delineation of natural risk zones in Greater Lisbon, Portugal. Journal of Risk Research, 18(5), 651-674. Repéré à https://www.academia.edu/7062391/Application_of_Social_Vulnerability_Index_SoVI_and_delineation_of_ natural_risk_zones_in_Greater_Lisbon_Portugal?auto=download

Mairie de Paris. (2017). Stratégie de résilience de Paris. Repéré à https://api-site-cdn.paris.fr/images/95335

Ministère des Affaires municipales et de l'Habitation (MAMH). (2010). Évaluation foncière : À quoi sert l'évaluation foncière municipale? Repéré à https://www.mamot.gouv.qc.ca/evaluation-fonciere/evaluation-fonciere-municipale-auquebec/a-quoi-ca-sert/

Ministère des Affaires municipales et de l'Occupation du territoire (MAMOT). (2014). Document à l'intention des citoyens : le rôle d'évaluation foncière et son contenu. Repéré à https://www.mamh.gouv.qc.ca/fileadmin/publications/ evaluation_fonciere/documentation/role_evaluation_contenu.pdf

Ministère de la Sécurité publique du Québec (MSP). (2018). Cadre pour la prévention de sinistres 2013-2022. Repéré à https://www.securitepublique.gouv.qc.ca/securite-civile/soutien-partenaires/soutien-aux-municipalites/cadre-pour-laprevention-de-sinistres.html

Ministère de la Sécurité publique du Québec (MSP). (2014). Politique québécoise de sécurité civile 2014-2024 : Vers une société plus résiliente aux catastrophes. Repéré à https://www.securitepublique.gouv.qc.ca/fileadmin/Documents/ securite_civile/publications/politique_2014-2024/politique_securite_civile_2014-2024.pdf 
Morin, M. (2008a). Concepts de base en sécurité civile. Québec: Ministère de la Sécurité publique. Repéré à https://www.securitepublique.gouv.qc.ca/fileadmin/Documents/securite_civile/publications/concepts_base/concepts_b ase.pdf

Morin, M. (2008b). Gestion des risques en sécurité civile. Québec: Ministère de la Sécurité publique. Repéré à https://www.securitepublique.gouv.qc.ca/fileadmin/Documents/securite_civile/publications/gestion_risques/gestion_ri sques.pdf

MRC de Coaticook. (2018). Règlement N. 6-25.1 : Règlement modifiant le règlement numéro 6-25 concernant le schéma d'aménagement et de développement durable sur le territoire de la Municipalité régionale de comté (MRC) de Coaticook. Repéré à https://www.mrcdecoaticook.qc.ca/Documentation/Reglement_625.1_aout\%202018_\%20FINAL.docx

Norris, F. H., Stevens, S. P., Pfefferbaum, B., Wyche, K. F. et Pfefferbaum, R. L. (2008). Community resilience as a metaphor, theory, set of capacities, and strategy for disaster readiness. American Journal of Community Psychology, 41(1-2), 127-150. Repéré à http://onlinelibrary.wiley.com/doi/10.1007/s10464-007-9156-6/full

Ouranos. (2018). Les inondations dans un contexte de changements climatiques. Repéré à https://www.ouranos.ca/publication-scientifique/FicheAvisInondation2018-Fr.pdf

Pigeon P. (2002). Réflexions sur les notions et les méthodes en géographie des risques dits naturels. Annales de Géographie, \#627-628, 29. Repéré à https://www.persee.fr/doc/geo_0003-4010_2002_num_111_627_21624

Serrhini, K. (dir.). (2009). La connaissance de la vulnérabilité indirecte du réseau de transport routier pour une meilleure gestion du risque d'inondation. Repéré à http://memoires.scd.univ-tours.fr/EPU_DA/LOCAL/2010PFE_Auroy _Dorceus_Sebirou.pdf

Tate, E., Cutter, S. L., \& Berry, M. (2010). Integrated multihazard mapping. Environment and Planning B-Planning \& Design, 37(4), 646-663. doi:10.1068/b35157. Repéré à http://journals.sagepub.com/doi/pdf/10.1068/b35157

Thomas, I., Bleau, N., Soto Abasolo, P., Desjardins-Dutil, G., Fuamba, M. et Kadi, S. (2012). Analyser la vulnérabilité sociétale et territoriale aux inondations en milieu urbain dans le contexte des changements climatiques, en prenant comme cas d'étude la Ville de Montréal. Montréal. Repéré à https://www.ouranos.ca/publicationscientifique/RapportThomasBleau2012_FR.pdf

Thomas, I. et coll. (2017). Analyse de la vulnérabilité aux inondations : Rapport public pour la ville de Saint-Raymond. Repéré à https://www.capsa-org.com/images/partenaires/cr/udem_rapport.pdf

Thomas, I. et Da Cunha, A. (2017). La ville résiliente : Comment la construire ? Montréal : Les Presses de l'Université de Montréal.

Thouret J. C. D’Ercole R. (1996). Vulnérabilité aux risques naturels en milieu urbain : effets, facteurs et réponses sociales. Cahier des Sciences humaines, 32(2), 407-422. Repéré à http://horizon.documentation.ird.fr/exldoc/pleins_textes/pleins_textes_4/sci_hum/010006241.pdf

Tricot, A. (2008). La prévention des risques d'inondation en France : entre approche normative de l'État et expériences locales des cours d'eau, Environnement Urbain, nº, p. 123-133. Repéré à http://www.vrm.ca/wpcontent/uploads/EUE2_Tricot.pdf

United Nations Office for Disaster Risk Reduction (UNISDR). (2015). Cadre d'action de Sendai pour la réduction des risques de catastrophe 2015-2030. Repéré à https://www.unisdr.org/files/43291_frenchsenda iframeworkfordisasterris.pdf 